\title{
Comparison of travelling ionospheric disturbance measurements with thermosphere/ionosphere model results
}

\author{
Ya. F. Ashkaliev ${ }^{1}$, G. I. Gordienko ${ }^{1}$, Ch. Jacobi ${ }^{2}$, Yu. G. Litvinov ${ }^{1}$, V. V. Vodyannikov $^{1}$, and A. F. Yakovets $^{1}$ \\ ${ }^{1}$ Institute of Ionosphere, Almaty, 480020, Kazakhstan \\ ${ }^{2}$ University of Leipzig, Institute for Meteorology, Stephanstr. 3, 04103 Leipzig, Germany
}

Received: 14 June 2002 - Revised: 5 September 2002 - Accepted: 18 September 2002

\begin{abstract}
Comparisons of modeled and measured responses of the ionosphere to the passage of atmospheric gravity waves are made for data recorded by an ionosonde located at Almaty $\left(76^{\circ} 55^{\prime} \mathrm{E}, 43^{\circ} 15^{\prime} \mathrm{N}\right)$ from June 2000 until May 2001. Temporal variations of the altitude $(h m \mathrm{~F})$ and electron content $(N m \mathrm{~F})$ of the F-layer peak are used for comparisons. A significant part of the observations showed well-defined wave structures on the $h m \mathrm{~F}, N m \mathrm{~F}$ and other parameter variations observed throughout the entire nights. Both the modeling study and measurements showed that, as the F-layer is lifted by the positive surge in gravity wave, the electron content at the F-layer peak decreases, with the slab thickness being increased as well. Subsequently, the opposite happens as $h m \mathrm{~F}$ falls below its equilibrium value. Some discrepancy between the model and experimental results related to the phase difference between $h m \mathrm{~F}$ and $N m \mathrm{~F}$ variations is revealed.
\end{abstract}

Key words. Ionosphere (ionosphere-atmosphere interaction, ionospheric disturbances)

\section{Introduction}

Internal atmospheric gravity waves (AGWs) play an important role in the dynamics of the middle and upper atmosphere. Propagating through the thermosphere and interacting with the ionospheric plasma, AGWs produce travelling ionospheric disturbances (TIDs). The propagation of AGWs in the neutral atmosphere and their ionospheric signature (TIDs) were studied both experimentally and theoretically for many years. Results of these studies are summarized in several review papers (Yeh and Liu, 1974; Hunsucker, 1982; Hocke and Schlegel, 1996). On the basis of theoretical and experimental investigations several models have been developed to describe the main characteristics of AGW-TID relationship on a global scale. These models reflect not only the level of understanding of the basic physical principles gov-

Correspondence to: A. F. Yakovets

(arthur@ionos.alma-ata.su) erning the atmospheric and ionospheric processes, but can be permanently improved by new experimental data incorporated in the model calculations. A comprehensive simulation of the relationship of all fundamental parameters (density, temperature, and wind velocity) of the neutral atmosphere perturbed by AGWs and all fundamental ionospheric parameters (electron density, ion and electron temperatures, magnetic field-aligned ion drift) has been performed recently by Kirchengast (1996). The large number of considered parameters makes the modeled results suitable, for example, for comparison with incoherent scatter data.

Comparison of ionosonde data with the modeled AGWTID relationship is more conveniently performed by simulation of the global atmospheric and ionospheric response to a single, short burst of enhanced electric field over a localised area at high-latitudes (Millward et al., 1993). This simulation is made on the basis of the global coupled thermosphere/ionosphere model (CTIM) that has been developed by scientists at the University of Sheffield and University College London (Fuller-Rowell et al., 1987). In this simulation the enhanced electric field causes enhancement of the auroral electrojet and, therefore, intense local Joule heating in the atmosphere. The expansion and subsequent relaxation of the local atmosphere generates large-scale AGWs propagating equatorwards and polewards from the event region. The waves propagate to mid-latitudes, creating TIDs on their path. The behaviour of the electron content $(N m \mathrm{~F})$ and altitude $(h m \mathrm{~F})$ of the F-layer peak, and the vertical profile of the electron content $\left(N_{e}(\mathrm{~h})\right)$ during the passing of AGWs were studied in this modelling. These parameters can be computed from experimental ionograms and the results of the groundbased vertical sounding of the ionosphere may be directly used to test the model. Thus, the objective of this paper is the experimental study of the response of the mid-latitude ionospheric F-layer to the passage of AGWs and comparison of that response with modeled results obtained by Millward et al. (1993). 

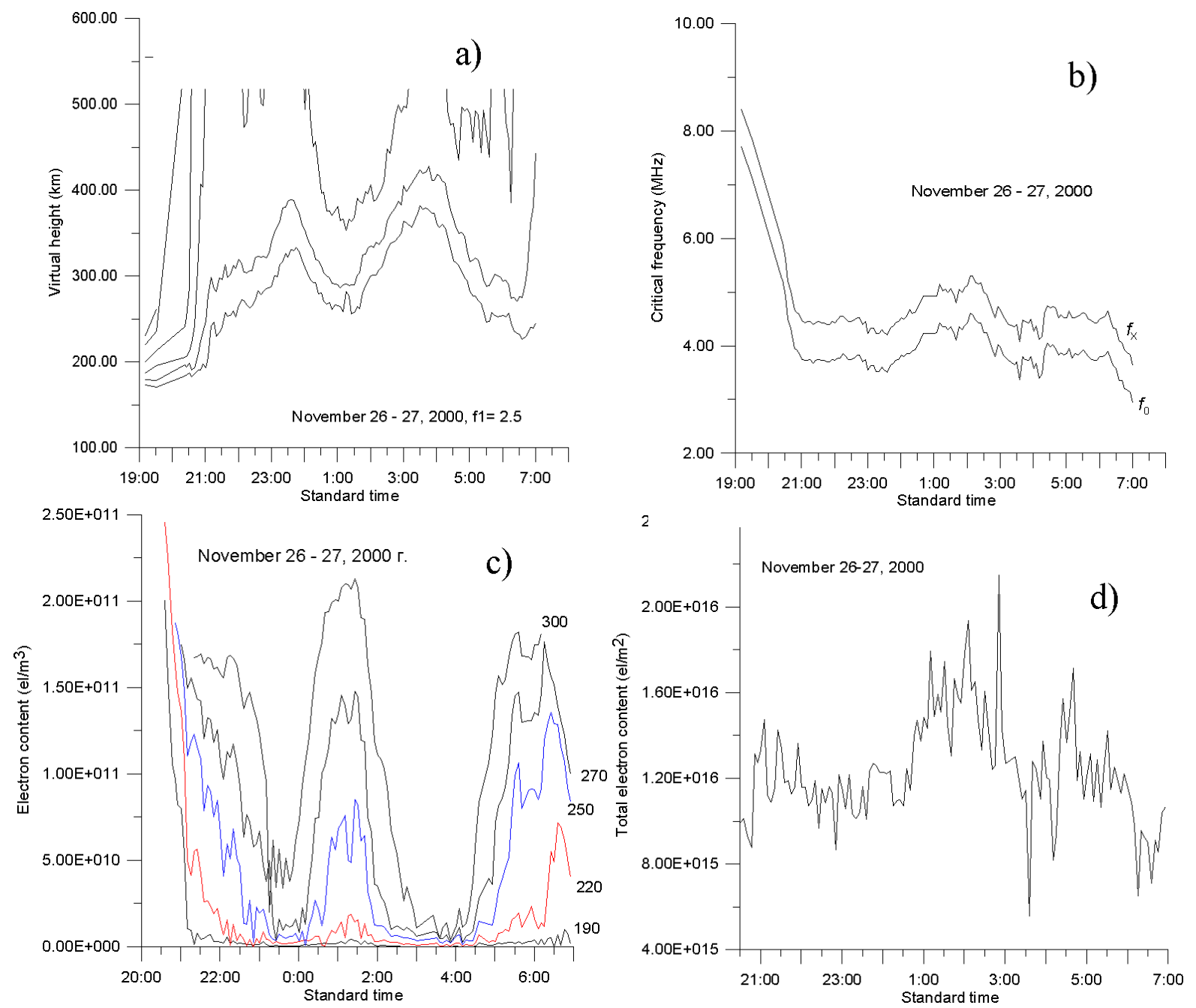

Fig. 1. Plots of the temporal variations of: (a) virtual heights ( $x$-component of the ionospheric signal) corresponding to specific frequencies of $2.5 \mathrm{MHz}, 3.5 \mathrm{MHz}, 4.5 \mathrm{MHz}, 5.5 \mathrm{MHz}$, and $6.5 \mathrm{MHz}$, (b) critical frequencies of the ordinary $\left(f_{o}\right)$ and extra-ordinary $\left(f_{x}\right)$ components, $(\mathbf{c})$ the electron content at series of heights $h=190 \mathrm{~km}, 220 \mathrm{~km}, 250 \mathrm{~km}, 270 \mathrm{~km}$, and $300 \mathrm{~km}$, (d) the total electron content below the F-layer peak.

\section{Temporal behaviour of ionospheric parameters}

For comparison CTIM model results and ionospheric vertical sounding night-time observations of TIDs in the F-region of the ionosphere were carried out from June 2000 through May 2001 by the digital multifunctional ionosonde BASIS installed at Almaty $\left(76^{\circ} 55^{\prime} \mathrm{E}, 43^{\circ} 15^{\prime} \mathrm{N}\right)$. The night-time of observations was chosen because a burst of the electric field was simulated in the night sector of the auroral region; in addition, the night-time mid-latitude ionosphere is characterised by large-scale TIDs with large amplitudes (Rice et. al., 1988; Hajkowicz, 1990; Yakovets et al., 1995). In general, the sounding was carried out during 8-10 nights per month. Ionograms were recorded every $5 \mathrm{~min}$. An observation run lasted about $12 \mathrm{~h}$ in winter and $8 \mathrm{~h}$ in summer, in order to eliminate periods of sunset and sunrise and to provide conditions of a quasi-stationary ionosphere.

The ionograms were analysed for virtual height $h^{\prime}(\mathrm{t})$, crit- ical frequency $f_{0} \mathrm{~F}(\mathrm{t})$, and $\mathrm{N}(\mathrm{h})$-profiles. BASIS provided an accuracy of $2.5 \mathrm{~km}$ for $h^{\prime}(\mathrm{t})$ and $0.05 \mathrm{MHz}$ for $f_{0} \mathrm{~F}(\mathrm{t})$. The parameters $h^{\prime}(\mathrm{t})$ and $f_{0} \mathrm{~F}(\mathrm{t})$ are directly available from the routine scaling of ionograms and, therefore, these parameters are frequently used for the analysis of large ionosonde data sets. In Figs. 1a and b temporal variations of $h^{\prime}(\mathrm{t}$ ) (for the extraordinary component of the reflected signal) and $f_{0} \mathrm{~F}(\mathrm{t})$, respectively, are shown for the night of 26-27 November 2000. This night was characterised by a high level of magnetic activity $\left(\Sigma K_{p}=28^{+}\right)$. The large magnetic storm with sudden commence began at 05:54 UT on 26 November 2000 and lasted for several days. These plots reveal periodical variations of the parameters considered. During a significant part of the measurements conducted, similar wave structures were visible in the $h^{\prime}(\mathrm{t})$ variations, observed throughout the entire night. However, corresponding $f_{0} \mathrm{~F}(\mathrm{t})$ variations were less clearly discernible, as one may see in Fig. 1. The amplitudes 


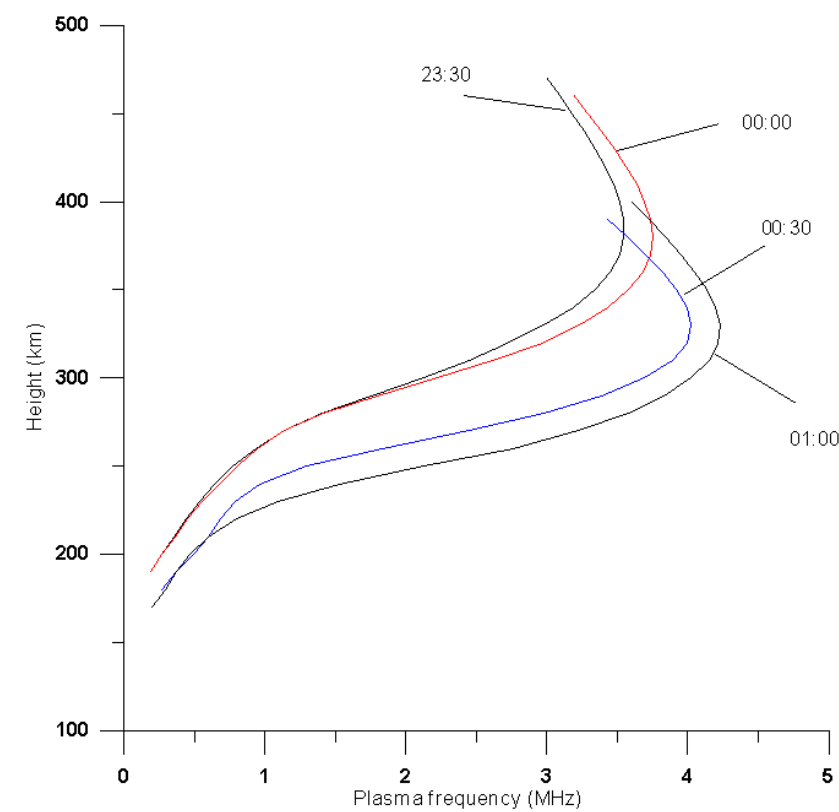

Fig. 2. Plasma frequency height profiles at 23:30, 00:00, 00:30, and 01:00 Almaty standard time for 26-27 November 2000.

of the $h^{\prime}(\mathrm{t})$ and $f_{0} \mathrm{~F}(\mathrm{t})$ variations changed from night to night, but these variations were almost in antiphase. From the set of $h^{\prime}(\mathrm{t})$ there is also evidence of downward phase propagation of observed waves. Millward et al. (1993) parameterised TIDs in the CTIM model by examining several quantities: $h m \mathrm{~F}, N m \mathrm{~F}$, and the vertical $\mathrm{N}(\mathrm{h})$-profiles. In order to compare model results and the present experimental results, we converted the ionograms into N(h)-profiles by using the accurate method of Titheridge (1985). Variations of the electron content at specific altitudes and total electron content below the F-layer peak were computed from N(h)-profiles. Examples are plotted in Figs. 1c and 1d, respectively. Figure 2 presents a sequence of the plasma frequency height profiles for the same observation on 26-27 November 2000. Taking into account that the plasma frequency $\left(f_{p}\right)$ is related to the electron content $N$ through $N=1.24 \cdot 10^{4} f^{2}$ (where $f$ is expressed in $\mathrm{MHz}$ and $N$ in el $/ \mathrm{cm}^{3}$ ), the plasma frequency height profiles can be easily transformed into $\mathrm{N}(\mathrm{h})$-profiles. Millward et al. (1993) have shown that AGWs generated by the auroral events can be considered as neutral atmosphere pressure waves, so that they may be directly characterised by changing heights of fixed pressure levels in the thermosphere. Considering plasma vertical oscillations when an AGW was passing overhead, Rishbeth (1995) concluded that the height of a certain "cell" of plasma with constant electron density tends to follow a level of constant atmospheric pressure. Thus, the virtual height variations of specific sounding frequencies may serve as a sensitive indicator of AGWs propagating through the thermosphere.

Comparing Figs. 1a and 2 it can be seen that the ionosphere is lifted by the positive pressure surge in the AGW, with the electron density in the F-layer peak being decreased and the F-layer thickness increased at the upper position of the F-layer. The informative picture of behaviour of the Flayer in the course of the AGWs passing over a measurement site can be obtained if temporal variations of the height of the F-layer peak $(h m \mathrm{~F})$ and its critical frequency $\left(f_{c r}\right)$ are plotted as a hodograph. Figure 3 shows typical examples of hodographs where adjacent points are separated by an interval of $30 \mathrm{~min}$ and arrows indicate the direction of running the process. Every hodograph presents several periods of the $h m \mathrm{~F}$ and $f_{c r}$ variations. In spite of their different forms, most hodographs have some common features. An irregular hodograph is shown in Fig. 3a. All other hodographs demonstrate elliptically polarized trajectories with clockwise rotation. The eccentricity of the ellipses varies from event to event (for example, hodographs shown in Fig. $3 \mathrm{c}$ are almost circularly polarized), but the tilt of the main axis lies in a narrow angular range. Figure $3 \mathrm{~d}$ shows the result of a superposition of the $h m \mathrm{~F}$ and $f_{c r}$ variations caused by passing AGWs and a linear trend of these parameters in the course of the measurements. The eccentricity of the ellipse and the tilt of the main axis are defined by a phase difference between $h m \mathrm{~F}$ and $f_{c r}$ variations and the ratio of variation magnitudes. Phase differences were estimated comparing the time series of $h m \mathrm{~F}$ and $f_{c r}$. Figure 4 shows the distribution of the phase difference. It is evident that practically all events fall into an interval of $100^{\circ}-180^{\circ}$, corresponding to a right-hand polarisation of hodographs. Such a result shows that $h m \mathrm{~F}$ and $f_{c r}$ variations are not in antiphase but the maximum in $h m \mathrm{~F}$ lags behind the minimum in $f_{c r}$, and the minimum in $h m \mathrm{~F}$ lags behind and the maximum in the $f_{c r}$. From Fig. 3 it is clear that there is a connection between amplitudes of $h m \mathrm{~F}$ and $f_{c r}$ variations. Figure 5 illustrates the relation between the peak-to-peak amplitudes of $h m \mathrm{~F}$ and $f_{c r}$ variations $(d h$ and $d f$ ). A general trend towards an increase of dh with df is seen, but the data points are scattered and the correlation coefficient only reaches 0.67 . However, in spite of considerable dispersion, there is the possible linear relationship $d h=19.8+45.1 \cdot d f$.

Recently, variations in total electron content (TEC) along the line of sight from a ground-based receiver to a GPS satellite have been studied. Therefore, it is of principal interest to compare these results with other techniques, and, therefore, to estimate whether TID can, in principle, be seen by satellite techniques as well. However, the data presented in this study do not allow one to measure TEC above the F-layer peak; only measurements below it are possible. However, it will be shown later that the F-region below the F-layer peak gives the main contribution to TEC variations caused by TID. Thus, to simulate TID measurements by a satellite, TEC below the F-layer peak was computed and the results are presented in Fig. 1d. It can be seen that the same periodic structure presents in the TEC record, but the long period variation $(T=4.7 \mathrm{~h})$ is hardly visible on the background of short period variations. The reason for that is as follows: from the delay of the same phase of TID between various altitudes we estimated a vertical wavelength of $\lambda_{z} \approx 1400 \mathrm{~km}$. This is large compared with the thickness of the F-layer, and thus, 

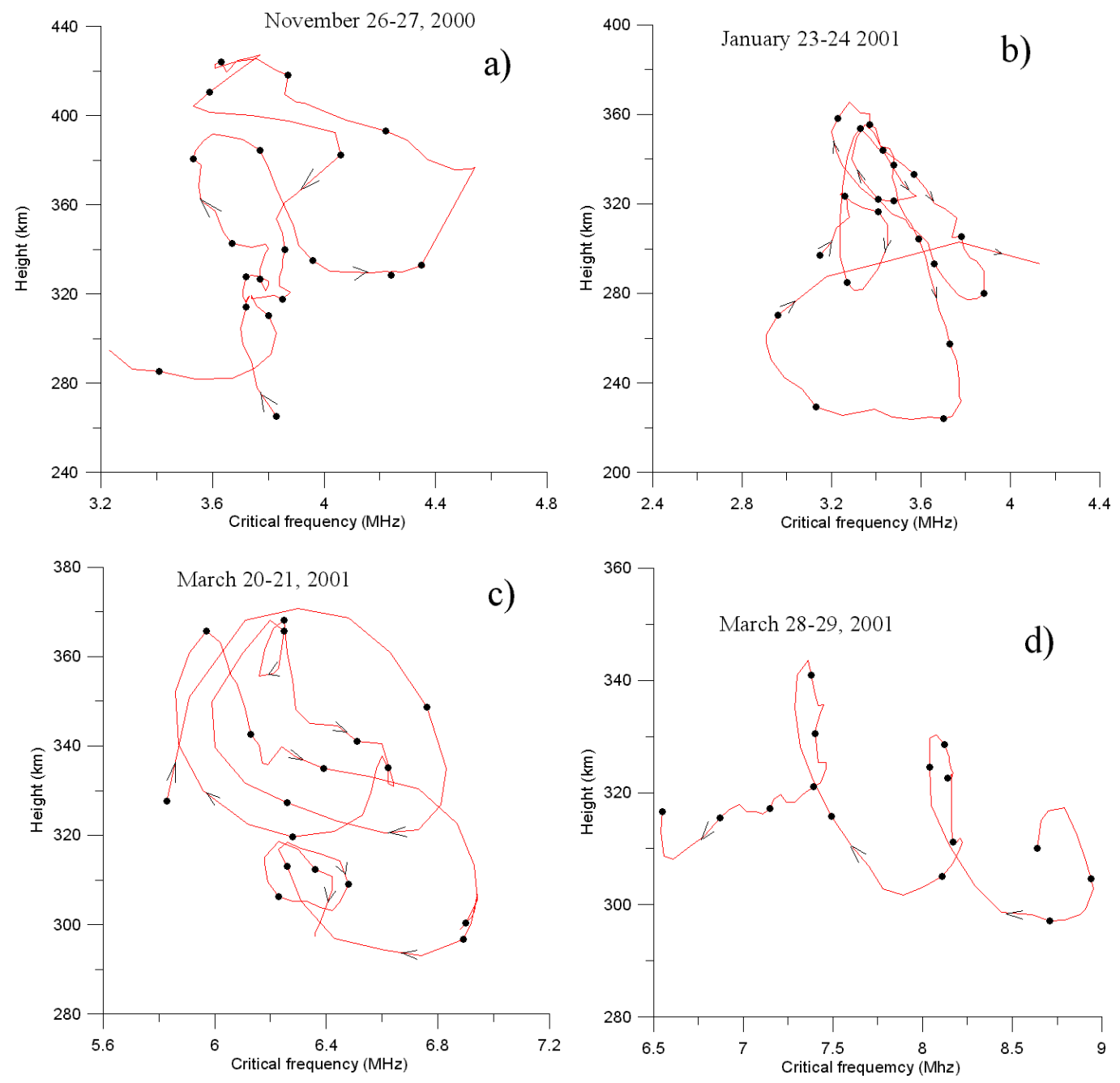

Fig. 3. Typical examples of hodograph show the time evolution of the $h m \mathrm{~F}$ versus $f_{c r}$.

the passage of the AGW lifts and descends the whole F-layer practically in phase. A small decrease in TEC in the upper part of the F-layer below the layer peak that should be caused by the decrease in the $N m \mathrm{~F}$ is compensated by increasing the layer thickness, leading to small variations of TEC.

\section{Wave amplitude height profiles}

It is known (Hines, 1960) that the upward energy flux of the AGWs remains constant in the nondissipative atmosphere. The wave amplitude increases with height, because the upward travelling wave propagates into a region of exponentially decreasing density of the atmosphere.

Clearly, this increase cannot be maintained indefinitely. At thermospheric altitudes, processes of dissipation, such as molecular viscosity, begin to compete with growth of the wave amplitude, leading to a maximum in the amplitude height profile. It seems that the altitude of the maximum amplitude of the AGW differs from that of the TID maximum because the electron content height profile superimposes on the AGW amplitude height profile. Thus, it is of great importance for the task of TID parameterisation to study height profiles of TIDs from measured ionograms.

For this purpose the 5-min interval series of the electron content height profiles were used. From these profiles the temporal behaviour of the electron content at specific heights separated with an interval of $10 \mathrm{~km}$ was computed, as in Fig. 1c. The wave amplitude, defined as half of the peakto-peak amplitude, was computed for each altitude. This allowed one to obtain the height profiles of the wave amplitude and its dependence on season and magnetic activity. The typical height profiles of the TID amplitude for three nights are plotted in Fig. 6. For one of the nights the variations of ionospheric parameters are already presented in Fig. 1. A total of 72 height profiles were analysed. The common shape of these height profiles can be approximated as a parabola, with its average thickness (defined at the level of 0.5 maximum amplitude) estimated as $\sim 60 \mathrm{~km}$. Figure 7 shows a distribution of an altitude of the TID's maximum amplitude. The altitude of the maximum amplitude is scattered between 200 


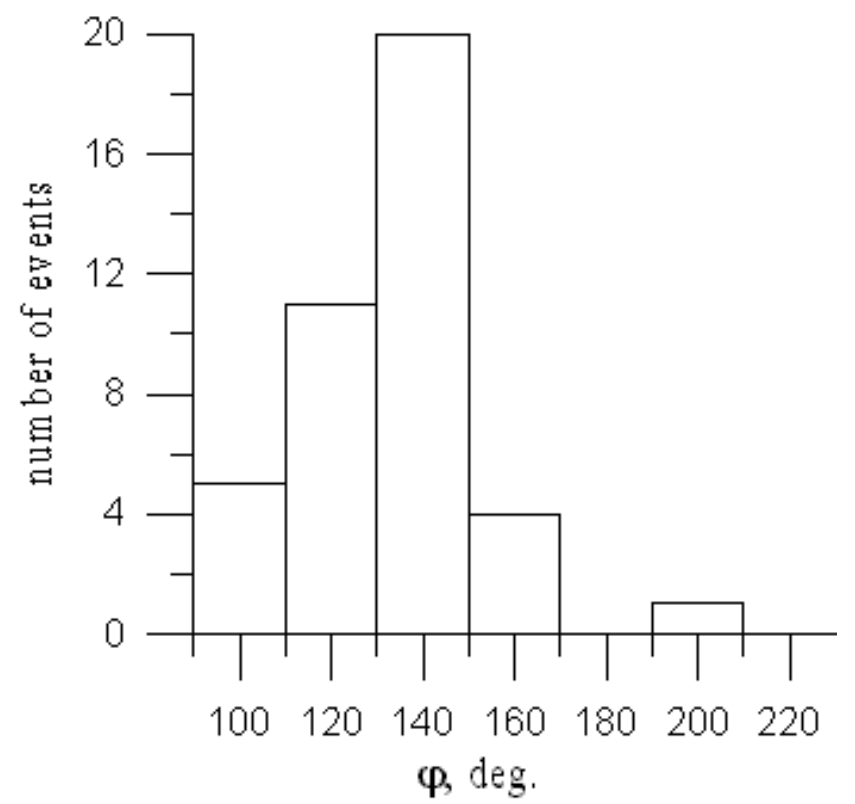

Fig. 4. Distribution of the phase difference between the $h m \mathrm{~F}$ and $f_{c r}$ variations.

and $300 \mathrm{~km}$, with the most probable value of $\sim 250 \mathrm{~km}$ (in $68.5 \%$ of all events the altitude is found between 220 and $260 \mathrm{~km})$. No evident seasonal dependence of the shape and position of the height amplitude profile were noticed. The weak dependence on the magnetic activity should be pointed out. During magnetic storms, there was a tendency for the maximum amplitude, its altitude, and the thickness of the height amplitude profiles to be larger than those for the quiet magnetic conditions, as can be seen from Fig. 4.

\section{Discussion}

Such behaviour of F-layer parameters is remarkably consistent with results of a model study (Millward et al., 1993). Their Figs. 6, 7, and 8 reveal the ionospheric response to the passing AGW. This modelled response looks like the experimental response presented here in Figs. 1, 2, and 3. Millward et al. (1993) have given an explanation of the physical processes taking place in the thermosphere and ionosphere, which were responsible for the F-layer behaviour in the course of the passing AGW over the site of observation.

The variations of the night-time ionosphere parameters during the AGW passing over the site of observation are principally caused by a redistribution of the plasma and are not due to changes in the production or loss processes. The redistribution of the plasma is caused by the motion of the ions along the magnetic field lines. The field-aligned velocity of ions can be expressed as $V\|=U\|+D \|$, where $U \|$ is the field-aligned component of the neutral wind caused by AGW. The diffusion term $D \|$ is the sum of the density gradient, temperature gradient, and gravity terms. Above $300 \mathrm{~km}$ the diffusion effect increases with height.

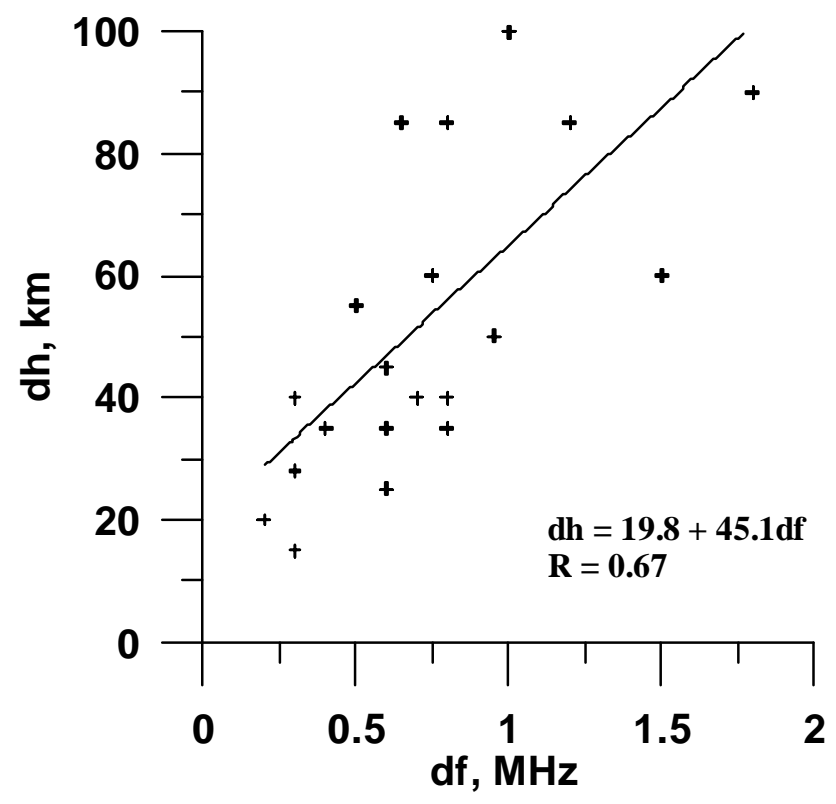

Fig. 5. Scatterplot of the peak-to-peak amplitudes of $h m \mathrm{~F}$ variations $(d h)$ versus those of $f_{c r}$ variations $(d f)$.

The ion flux height profiles plotted in Fig. 7 of Millward et al. (1993) indicate the redistribution of plasma that occurs due to effects described above. Before the arrival of the AGW, a small downward ion flux from the plasmasphere contributes to maintaining the night-time F2-layer by providing a small, continuous source of new ionisation, which compensates for the continuous loss of ionisation through recombination and diffusion. The ion flux is strongly modified by the arriving wave. Maximum upward flux occurs near the time of the crest of the height variation wave, producing an increase in $h m \mathrm{~F}$. The maximum flux, in this plot, lies at an altitude above the F-layer peak. Also, it can be seen that the magnitude of the flux gradient, along the magnetic field lines, is greater above the flux peak than below it. This divergence in the field-aligned flux is responsible for the decrease in $N m \mathrm{~F}$, which reaches a minimum near the time of a maximum in $h m F$. Downward ion flux of similar magnitude and similar divergence leads to a downward shift of the location of the electron density profile. It is necessary to point out some discrepancy between the experimental and modeled results related to the phase difference between $N m \mathrm{~F}$ and $h m \mathrm{~F}$ variations. If modeled results show very small difference between moments of minimum in $N m \mathrm{~F}$ and maximum in $h m \mathrm{~F}$, then the experimental phase difference between these moments varies in a rather broad range from one night to another one. A reason for the delay of the maximum in $h m \mathrm{~F}$ relative to the minimum in $N m \mathrm{~F}$ is not yet understood.

The fact that the model study was performed for a single burst of electric field enhancement, while experimental records of TIDs commonly demonstrate a sequence of quasiperiodical variations of ionospheric parameters does not confine the results of comparison, because in practice, it is com- 


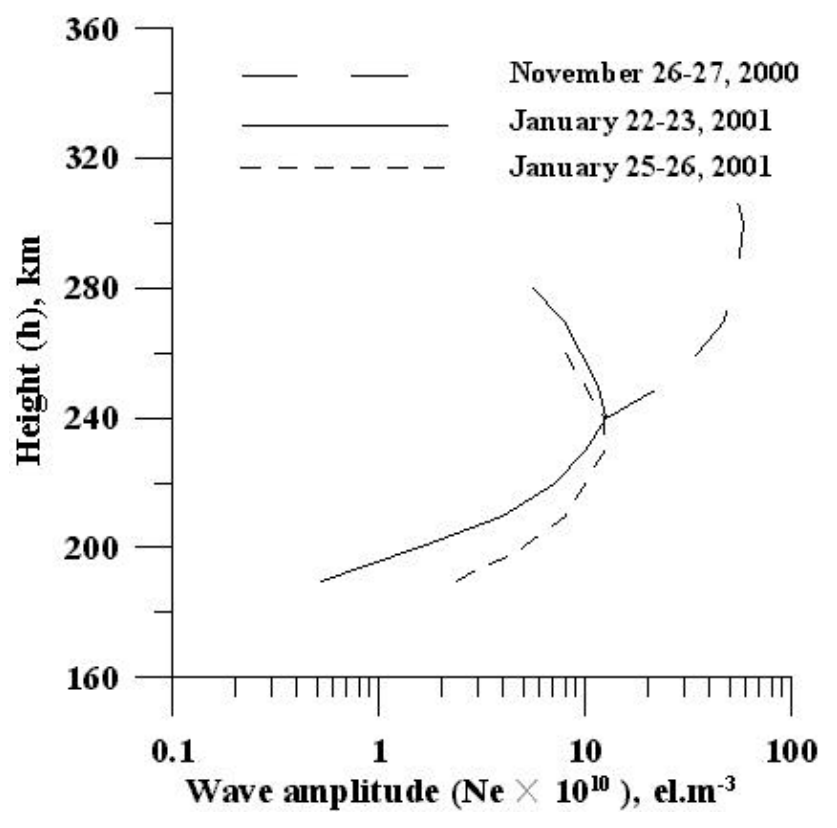

Fig. 6. Wave amplitude height profiles for three nights with different magnetic activity: 26-27 November $2000\left(\Sigma K_{p}=28\right) ; 22-23$ January $2001\left(\Sigma K_{p}=22\right) ; 25-26$ January $2001\left(\Sigma K_{p}=23\right)$.

mon to observe several periodical consecutive bursts in the electric field.

Thus, this study shows that the F-layer behaviour during the passage of the AGW is consistent with the results of a model study of the atmospheric and ionospheric response to a burst of enhanced electric field at high latitudes.

\section{Conclusions}

An experimental study of the response of the mid-latitude night-time ionosphere F-layer to passing atmospheric gravity waves was carried out. Ionograms were analysed for temporal variations of virtual heights taken for several fixed frequencies reflected in the ionosphere (the virtual heights of the constant electron content), critical frequency of the ionosphere F-layer, and height profiles of electron content $(\mathrm{N}(\mathrm{h})$-profiles). A significant part of the observations showed well-defined wave structures on the $h^{\prime}(\mathrm{t})$ variations observed throughout the entire night, but the corresponding $f_{0} \mathrm{~F}(\mathrm{t})$ variations were less discernible. These $h^{\prime}(\mathrm{t})$ and $f_{0} \mathrm{~F}(\mathrm{t})$ variations were almost in antiphase. From the $h^{\prime}(\mathrm{t})$ there is evidence of downward phase propagation of the observed waves. The temporal behaviour of the electron content at a series of specific heights allowed one to obtain the height profiles of the wave amplitude $\left(\mathrm{el} / \mathrm{m}^{3}\right)$. The common shape of the height profiles can be approximated by a parabola with an average thickness of $\sim 60 \mathrm{~km}$. No evident seasonal dependence was visible, and the dependence on magnetic activity was weak. During magnetic storms, there was a tendency for the maximum amplitude, its altitude, and the thickness of the height amplitude profiles to be larger than those under the

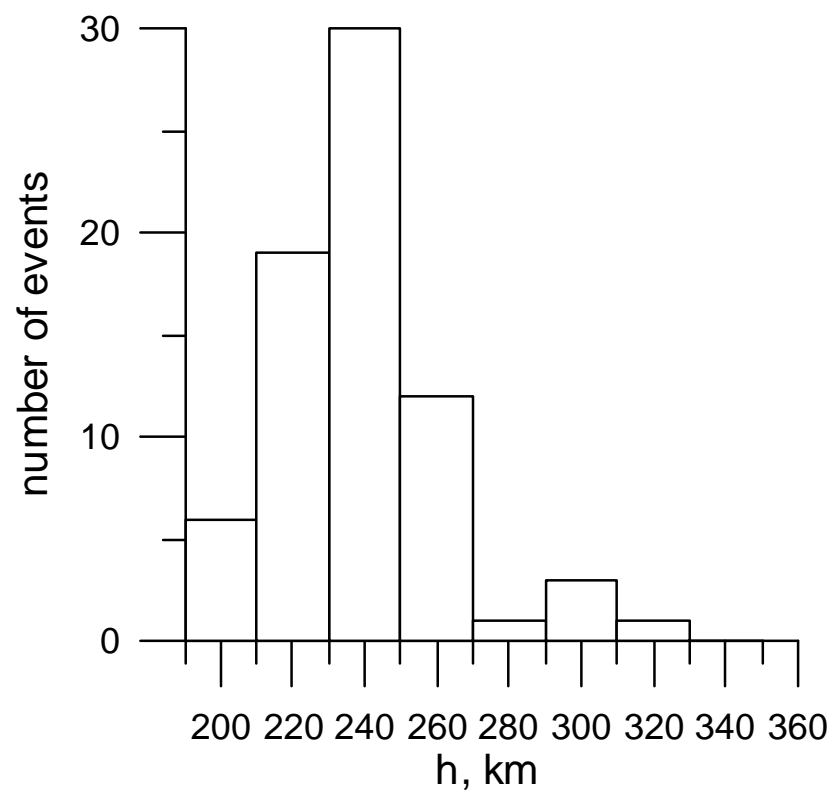

Fig. 7. A distribution of an altitude of the TID's maximum amplitude.

quiet magnetic conditions. A consequence of N(h)-profiles calculated for a period of the passing wave showed them to move up and down in phase with the passing wave. As the F-layer is lifted by the positive surge in gravity wave, the electron content at the F-layer peak decreases, with the slab thickness being increased as well. Subsequently, the opposite happens as $h m \mathrm{~F}$ falls below its equilibrium value. Such characteristics of the F-layer behaviour is remarkably consistent with results of a model study of the atmospheric and ionospheric response to a short burst of enhanced ion convection at high latitudes performed with the CTIM model.

Acknowledgements. This work was performed with support of INTAS through grant No. 991-1186.

Topical Editor M. Lester thanks R. Hunsucker and M. Denton for their help in evaluating this paper.

\section{References}

Fuller-Rowell, T. J., Rees, D., Quegan, S., Moffett, R. J., and Bailey, G. J.: Interactions between neutral thermospheric composition and the polar ionosphere using a coupled ionospherethermosphere model, J. Geophys. Res., 92, 7744-7775, 1987.

Hajkowicz, L. A.: Global study of large scale travelling ionospheric disturbances (TIDs) following a step-like onset of auroral substorm in both hemispheres, Planet. Space Sci., 38, 913-923, 1990.

Hines, C. O.: Internal atmospheric gravity waves at ionospheric heights, Can. J. Phys., 38, 1441-1481, 1960.

Hocke K. and Schlegel, K.: A review of atmospheric gravity waves and travelling ionospheric disturbances: 1982-1995, Ann. Geophysicae, 14, 917-940, 1996. 
Hunsucker, R. D.: Atmospheric gravity waves generated in the high-latitude ionosphere: A review, Rev. Geophys., 20, 293-315, 1982.

Kirchengast, G.: Elucidation of the physics of the gravity wave-TID relationship with the aid of theoretical simulations, J. Geophys. Res., 101, 13 353-13 368, 1996.

Millward, G. H., Moffet, R. J., Quegam, S., and Fuller-Rowell, T. J.: Effect of an atmospheric gravity wave on the midlatitude ionospheric F-layer, J. Geophys. Res., 98, 19 173-19 179, 1993.

Rice, D. D, Hunsucker, R.D., Lanzerotti, L. J., Crowly, G., Williams, P. J.S., Craven, J.D., and Frank, L.: An observation of atmospheric gravity wave cause and effect during the Octo- ber 1985 WAGS campaign, Radio Sci., 23, 919-930, 1988.

Rishbeth, H., Jenkins, B., and Moffett, R. J.: The F-layer at sunrise, Ann. Geophysicae, 13, 367-374, 1995.

Titheridge, J.E.: Ionogram analysis with the generalised program Polan, National Geophysical Data Center, Boulder, CO USA, p. 189, 1985.

Yakovets A. F., Drobjev, V. I., and Litvinov, Yu. G.: The spatial coherence of travelling ionospheric disturbances at mid-latitudes, J. Atmos. Terr. Phys., 57, 25-33, 1995.

Yeh K.C. and Liu, C. H.: Acoustic-gravity waves in the upper atmosphere, Rev. Geophys. Space Phys., 12, 193-216, 1974. 